\title{
Photonics for Coherent MIMO Radar: an Experimental Multi-Target Surveillance Scenario
}

\author{
S. Maresca ${ }^{1}$, G. Serafino ${ }^{1}$, F. Scotti ${ }^{2}$, F. Amato ${ }^{1}$, L. Lembo ${ }^{1,3}$, A. Bogoni ${ }^{1,2}$, P. Ghelfi ${ }^{2}$ \\ ${ }^{1}$ TeCIP Institute, Scuola Superiore Sant'Anna, Pisa, ITALY \\ email: salvatore.maresca@sssup.it
}

${ }^{2}$ PNTLab, Consorzio Nazionale Interuniversitario per le Telecomunicazioni (CNIT), Pisa, ITALY

${ }^{3}$ Vallauri Institute, Centro di Supporto e Sperimentazione Navale (CSSN), Livorno, ITALY

\begin{abstract}
This paper investigates the target detection and localization capabilities of a coherent multiple input multiple output (MIMO) radar network designed and implemented using photonic technology. The benefit offered by photonics is twofold: it guarantees longtime phase stability and frequency/phase coherence between the transmitted and received radio frequency signals; secondly, it allows remoting the antennas by exploiting optical fibers. The proposed radar network demonstrator, which is composed of two transmitting and two receiving antennas in the $X$-band with $100 \mathrm{MHz}$ signal bandwidth, operates in a real down-scaled outdoor scenario for detecting two collaborative closely-spaced moving targets. The preliminary results demonstrate the effective impact of photonics applied to coherent centralized radar networks and provide some guidelines for the development of more complex and application-tailored radar networks.
\end{abstract}

\section{Introduction}

Directed by the rapid changes in national and international assets, the ever-increasing demand for radar-based surveillance applications pushes towards the necessity of "sensor fusion". In other words, standalone radars are no more sufficient to meet all the operative requirements. However, "classical" multistatic radars employing multiple closely and/or widely separated transmitters (TXs) and receivers (RXs) in the same area, seldom cooperate with each other. Rather, mostly due to implementation costs and sensor deployment constraints (e.g. low or no availability of high bandwidth long-range communication links between the remote nodes), they typically provide multiple uncorrelated information of the same scene (e.g. target detections, tracks) to a master node for data fusion (i.e. decentralized processing). This solution simplifies the synchronization procedure and reduces the amount of data to be shared, leading to a significant performance limitation due to the unavoidable information loss determined by this simplification.

In such a context, centralized multiple-input multiple-output (MIMO) radars can rely on a better coordination among the network nodes [1]. Their TXs can employ different waveforms, while their RXs can process signals coming from the multiple widely distributed emitters. The increased spatial information improves target detection and localization performance, especially when the target exhibits a low radar cross section (RCS), or when the RCS is characterized by a high angular variability. Unfortunately, coherent MIMO radars with widely separated antennas require precise signal synchronization and reliable large-bandwidth longrange signal distribution among the network nodes. Up to now, these two issues have represented the main limitation factor to the development of such systems in real operative scenarios. 
Fortunately, in the last two decades, microwave photonics has matured to the point of being considered an ideal candidate for the development of coherent MIMO radar networks with widely distributed antennas, as conjectured in [2]. The benefit offered by photonics is twofold: it guarantees long-time phase stability and frequency/phase coherence between the transmitted and received radio frequency (RF) signals; secondly, it allows remoting the antennas by exploiting optical fibers. In photonics-based radars, signal generation, distribution and reception leverage on the unprecedented frequency flexibility and phase noise stability guaranteed by photonics [3]. Additionally, signal distribution by means of optical fibers assures high-quality and high-coherence, as well as the needed large bandwidth connectivity between the radar nodes [4]. The first coherent photonics-based radar system [5] and the promising results presented in [6] pushed the scientific community to develop the first photonics-based radar network demonstrators in both laboratory [7], [8] and outdoor environments [9].

In this paper, we further investigate the network presented in [9], by testing it in a real outdoor scenario with multiple closely-spaced targets. The network is composed of two TXs and two RXs, which are connected to a photonic core through optical fibers. At the actual status of development, the photonic core generates a $100 \mathrm{MHz}$-bandwidth linear frequency modulated (LFM) upsweep chirp signal at $9.7 \mathrm{GHz}$ (X-band). The orthogonality between the two transmitted waveforms is achieved by means of time domain multiplexing (TDM). In the infield experiment four low-gain/low-directivity horn antennas are distributed along a baseline of about $20 \mathrm{~m}$. As it will be discussed, the cooperative targets, i.e. two small metallic cylinders carried by two commercial mini-drones, can be correctly resolved even when moving at close distance, considerably improving the cross-range resolution of the system. These results confirm the validity of the proposed architecture, demonstrating that the achieved cross-range resolution provided by the coherent MIMO processing is far better than the one guaranteed by non-coherent processing, although the dataset is affected by antenna cross-talk, multipath propagation, clutter disturbance, and RF interference.

This paper is organized as follows. In Section 2 we describe the proposed photonic radar network architecture; in Section 3, we outline the digital signal processing (DSP) chain, while in Section 4 we present the experimental setup and discuss the preliminary results. Finally, in Section 5 we summarize the work and we draw the conclusions.

\section{Architecture of the Photonic Radar Network}

The proposed radar network consists of a photonic core, a colocated acquisition system, and two radar heads (RHs) with one TX and one RX each, that can be opportunely remoted by means of optical fibers, as depicted in Fig. 1 and described in [9]. The photonic core develops around the optical master clock, which is implemented by a solid-state mode-locked laser (MLL). The MLL is a pulsed laser, whose optical spectrum is a comb of several phase-locked lines, spaced by the pulses repetition frequency $f_{\mathrm{MLL}}=400 \mathrm{MHz}$. This feature allows generating RF carriers with extremely low phase noise $(\mathrm{PN})$, upon the opto-electronic $(\mathrm{O} / \mathrm{E})$ conversion of the MLL laser in a photodiode (PD) [3], thus implementing high-quality RF up-conversion and down-conversion. Moreover, exploiting a single MLL for these operations ensures a perfect synchronization of the RHs and maintains the coherence of the signals, which is the most crucial issue in coherent MIMO radars. In the case of up-conversion, the radar signal, which is digitally generated at intermediate frequency (IF), is first modulated on the MLL spectrum by an electrooptic modulator, and then photodetected, generating many replicas each centered at $k \cdot f_{\mathrm{MLL}} \pm \mathrm{IF}$, with $k$ positive integer [5], [6]. The desired RF output carrier frequency $f_{\mathrm{RF}}$ can be selected by means of a microwave filter, centered at $f_{\mathrm{RF}}$ and with bandwidth larger than $B$, where $B$ is the desired signal bandwidth. In down-conversion, the RF signal is similarly modulated on the MLL, then the $\mathrm{O} / \mathrm{E}$ conversion at the PD generates replicas of the RF signal down-converted at 


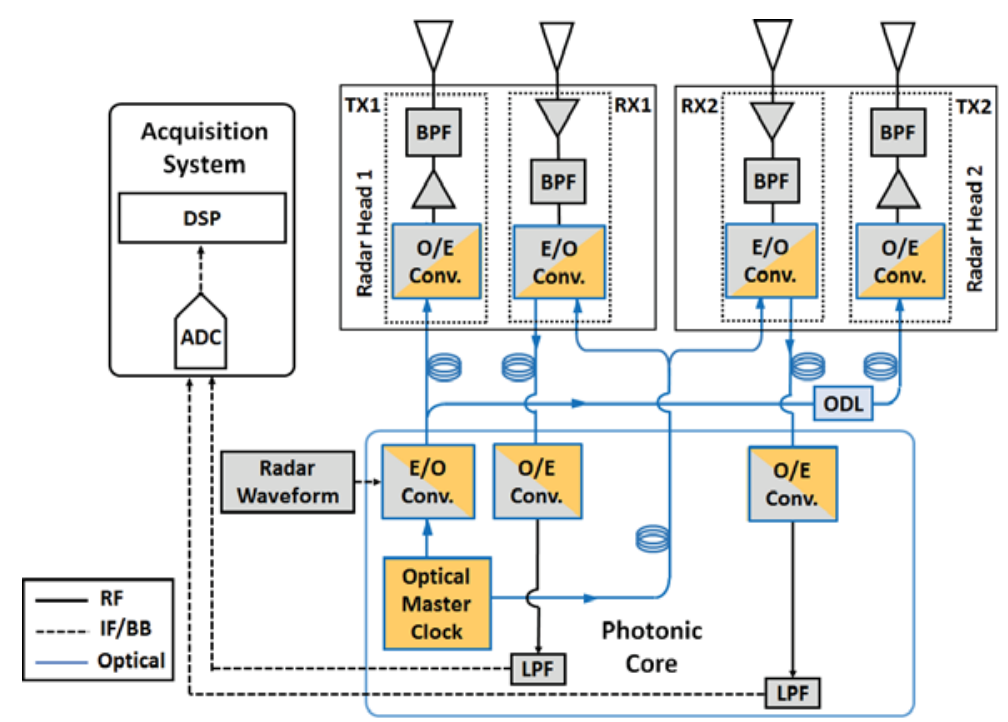

Figure 1: Architecture of the proposed Photonic Radar Network. DSP: Digital Signal Processing; ADC: Analog-to-Digital Converter; RF: Radio Frequency; LPF: Low-Pass Filter; E/O: Electro-Optical; ODL: Optical Delay Line; O/E: Opto-Electrical; IF/BB: Intermediate Frequency/Base Band; BPF: Band-Pass Filter.

$k \cdot f_{\mathrm{MLL}} \pm \mathrm{IF}$, including the IF (for $k=0$ ), the closest to baseband (BB) [5]. The large MLL optical spectrum ensures high efficiency, and the wide $\mathrm{E} / \mathrm{O}$ and $\mathrm{O} / \mathrm{E}$ bandwidth of the available modulators and PDs allows for the flexible management of RF signals up to several tens of $\mathrm{GHz}[3]$.

As reported in Fig. 1, the optical master clock feeds the E/O conversion of the radar waveform in transmission as well as of the received signal. The connections between the photonic core and the TXs/RXs at the RHs are realized through spans of single-mode fiber (SMF), as depicted by the coils in Fig. 1, which grant small propagation losses, absence of electromagnetic interference, and preservation of the signal coherence even with large signal bandwidth [4]. Once the E/O converted radar waveform is delivered to a TX, this operates its $\mathrm{O} / \mathrm{E}$ conversion which implies the up-conversion of the IF signal to RF. Here, the radar signal is generated at IF frequency $f_{\mathrm{IF}}=100 \mathrm{MHz}$. Thus, after proper amplification and band-pass filtering, the radar signal is transmitted by the antenna. The employed band-pass filters (BPFs) are multi-cavity filters with $-3 \mathrm{~dB}$ bandwidth of $100 \mathrm{MHz}$ and centered at $f_{\mathrm{RF}}=9.7 \mathrm{GHz}$. The employed antennas at the TXs are ultra-wideband Vivaldi-shaped horn antennas with about $50^{\circ}$ half power beam width (HPBW) aperture and $12 \mathrm{dBi}$ maximum gain. The optical delay line (ODL), consisting of a $1 \mathrm{~km}$-long SMF spool inserted just before the TX of RH2, implements the TDM between the two RHs. The RXs are equipped with antennas similar to those of the TXs. The detected radar echoes received by the RHs antennas are amplified, pass-band filtered and E/O converted. Then, the received signals are transmitted back to the photonic core and $\mathrm{O} / \mathrm{E}$ converted. After this operation, the signals from each RX are low-pass filtered and fed into a two-channel acquisition system, where they are digitized by an analog-to-digital converter (ADC) at 400 MS/s per channel.

\section{Digital Signal Processing Scheme}

Considering the general case of a radar network with $M$ TXs and $N$ RXs, the photonic core shall have $N$ ADCs digitizing the signals received by the RXs, each of these signals being the combination of the $M$ echoes from each of the TXs, down-converted at $f_{\text {IF. }}$ In the following, we will indicate with the subscript $k, l$ the generic bistatic pair, i.e., the signal determined by the $k^{\text {th }}$ TX and $l^{t h} \mathrm{RX}$, for $k=1, \ldots, M$ and $l=1, \ldots, N$. In the preliminary signal conditioning stage [10], the $N$ digitized data streams are split into the individual bistatic channels for data 


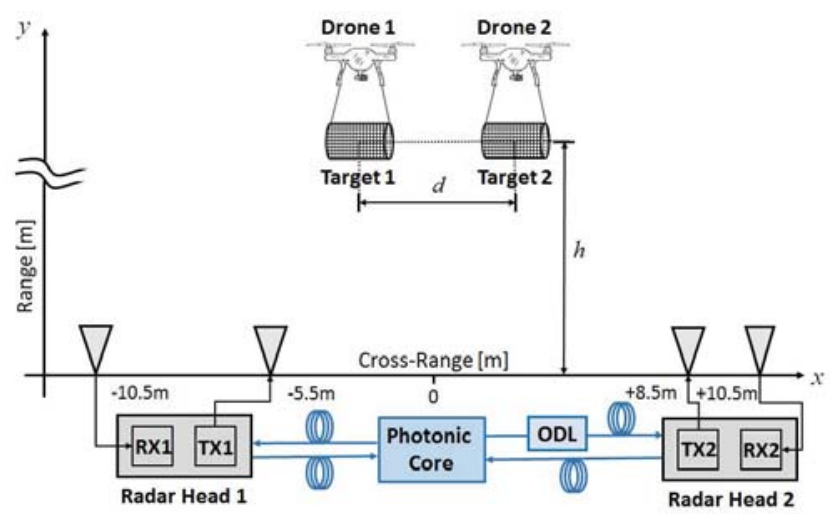

Figure 2: Geometry of the coherent photonics-based MIMO radar network and in-field experimental setup.

processing. To maximize the signal-to-noise ratio (SNR), the $M \times N$ channels are filtered at IF and then down-converted to BB. Pulse compression (i.e., matched filtering) is performed on the received pulsed signals. For the sake of brevity, we skip the details of these operations, which can be found in [9], [10]. The proposed radar network architecture ensures excellent phase coherence, because the same optical clock is used first in transmission for the up-conversion and then at the receiver for the down-conversion, for all the radar signals. Moreover, the optical oscillator stands out for its low PN curve [3]. As reported in [11], if the angular jitter of the architecture is lower than $10^{-1} \mathrm{rad}$, the effects on MIMO coherent detection performance are negligible. Otherwise, phase jitter could negatively affect not only target detection performance, but also increase the sidelobe level, thus introducing false alarms. The temporal jitter of the considered system architecture (i.e., the integration of the oscillator PN for offset frequencies in the interval $[20 \mathrm{~Hz}, 200 \mathrm{MHz}]$ ) is in the order of $10^{-12} \mathrm{~s}$, while the angular jitter is in the order of $10^{-2} \mathrm{rad}$, far better than the limit identified in [11]. For this reason, coherent MIMO processing becomes possible. Under the assumption that both additive noise (AN) and PN terms are present, the signal received by the $l^{\text {th }} \mathrm{RX}$, is [12]:

$$
r_{k, l}(t)=a_{k, l} \cdot s_{k}\left(t-\tau_{k, l}(x, y)\right) e^{j\left[\theta\left(t-\tau_{k, l}(x, y)\right)-\theta(t)\right]}+n_{k, l}(t),
$$

where $s_{k}(t)$ is the signal transmitted by the $k^{\text {th }} \mathrm{TX}, a_{k, l}$ is an amplitude factor, while $\tau_{k, l}(x, y)$ is the time delay proportional to the bistatic distance, which is function of the target location $(x, y)$ and on the $k^{\text {th }} \mathrm{TX}$ and $l^{\text {th }} \mathrm{RX}$ positions in the Cartesian plane. The term $n_{k, l}(t)$ represents the AN, generally modelled as an additive white Gaussian noise (AWGN) stochastic process. The term $\theta(t)$ takes into account the phase shift caused by the oscillator instability. If the angular jitter is below the $0.1 \mathrm{rad}$ limit, the PN detrimental influence on the signal is negligible. Therefore, the following log-likelihood function suitable for a MIMO approach can be used [12]:

$$
\ln [f(r(t) \mid(x, y))]=c^{\prime} \cdot\left|\sum_{k=1}^{M} \sum_{l=1}^{N} e^{-j 2 \pi f_{I F} \tau_{k, l}(x, y)} \int r_{k, l}^{B B *}(t) \cdot s_{k}^{B B}\left(t-\tau_{k, l}(x, y)\right) d t\right|+c^{\prime \prime} .
$$

According to eq. (2), for each possible target location with coordinates $(x, y)$ in the Cartesian search space, the decision statistic is computed determining for all the $M$ TXs and $N$ RXs the correlation between the received and transmitted $\mathrm{BB}$ signals. Moreover, a phase term compensates the phase shift due to the time delay $\tau_{k, l}(x, y)$ and to the TX-RX pair. After rephasing each term, all the $M \times N$ correlation contributes are summed together coherently. For further details, see [12].

\section{Experimental Setup and Results}

In our experiment, the radar waveform is a LFM pulse with $100 \mathrm{~ns}$ duration, pulse repetition interval (PRI) of $50 \mu \mathrm{s}$ and $B=100 \mathrm{MHz}$ centered at $f_{\mathrm{RF}}=9.7 \mathrm{GHz}$, with a monostatic range 


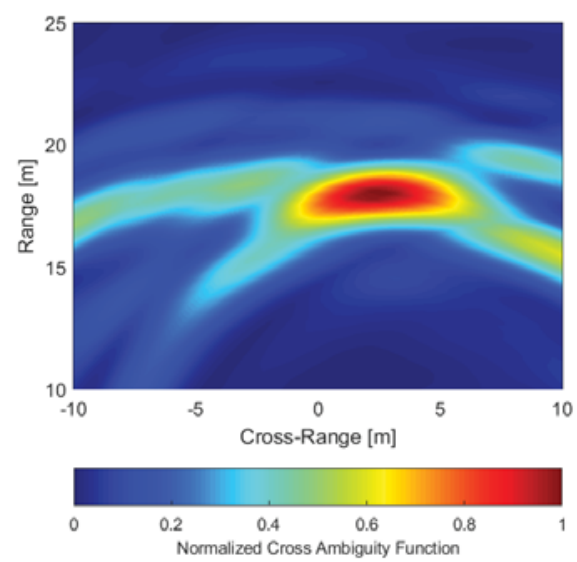

(a)

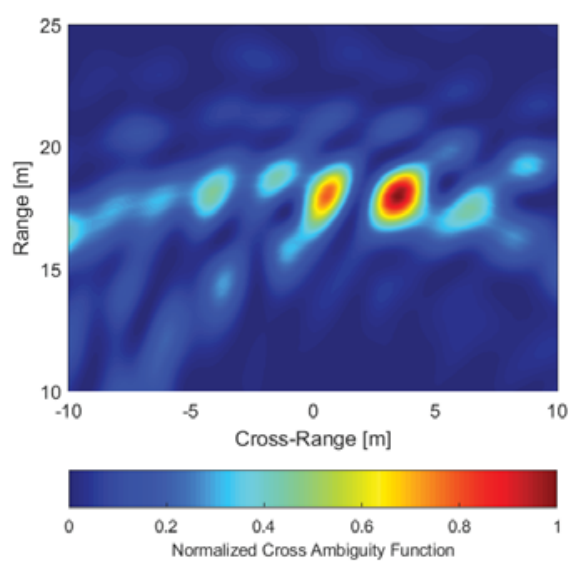

(b)

Figure 3: Non-coherent (a) versus coherent MIMO processing output in the proposed multi-target scenario.

resolution of $1.5 \mathrm{~m}$. The proposed coherent radar network is tested outdoor, on the rooftop of our lab. The network is deployed, according to the scheme in Fig. 2, with the four antennas aligned over a $21 \mathrm{~m}$-long baseline. These are oriented upwards, to mitigate clutter and multipath returns due to surrounding structures, buildings and vegetation, and to ensure the simultaneous illumination of both targets. The output power from each TX antenna is $100 \mathrm{~mW}$. The targets consist of two cylinders, with $17 \mathrm{~cm}$ radius and $50 \mathrm{~cm}$ height, made of a tight-mesh metal net and hanging from two mini-drones. The height of the two targets was controlled and kept always between 15 and $20 \mathrm{~m}$, while the two drones were hovering at the same height $h=18 \mathrm{~m}$ at about a distance $d=3 \mathrm{~m}$.

The experiment is realized with a scaled-down geometry. However, the presence of a long spool of fiber (the ODL in Fig. 1) simulates a distance of about $1 \mathrm{~km}$ between the two RHs. In the following, the results will demonstrate the low attenuation and negligible phase distortion introduced by the fiber. Moreover, we can consider the network being characterized by widely distributed antennas, since the four channels are spatially decorrelated, according to the definition proposed in [1]. Although the demonstration is straightforward, we omit it for brevity. Before evaluating the decision statistics (i.e. the normalized cross ambiguity functions), the stream acquired by the ADC is digitally filtered with an ideal BPF with $100 \mathrm{MHz}$ bandwidth centered at $f_{\mathrm{IF}}$. Then, the signal is down-converted to $\mathrm{BB}$ and the four channels are extracted. The fused signal output is evaluated over a $25 \mathrm{~ms}$ integration time (i.e. 500 PRIs). Then, both the non-coherent and coherent MIMO processing outputs are evaluated in a search space of $151 \times 201$ samples in the interval $[10,25] \mathrm{m}$ and $[-10,10] \mathrm{m}$ (i.e. with $10 \mathrm{~cm}$ spacing), along range and cross-range, respectively. Results are depicted in Fig. 3(a) and 3(b), respectively.

As we can observe in Fig 3(a), the two targets are too close to be correctly separated in the crossrange with a standard non-coherent processing. Indeed, being the in-range distance from the baseline of the two targets as $18 \mathrm{~m}$, and given the antenna HPBW, the expected monostatic crossrange resolution is $15.7 \mathrm{~m}$. The presence of high sidelobes in the monitored area is due not only to the presence of clutter and multipath, but also to the reduced MIMO configuration (i.e. few TXs and RXs). In this sense, a larger number of TXs and RXs would significantly reduce the intensity of the peaks and grant a finer cross-range resolution. Fig. 3 (b) depicts instead the output of the coherent MIMO processing case. Here, the two targets are correctly separated in crossrange, around $3 \mathrm{~m}$ apart from each other. Thus, the cross-range resolution has been improved by a factor of 5. Unfortunately, the drones RCS is too small to be able to detect them.

Given the reduced MIMO configuration and the non-idealities introduced by the environment and target RCS fluctuations, the results obtained in terms of cross-range resolution are truly remarkable. Despite the very preliminary setup, such results demonstrate the feasibility of the 
proposed architecture and the decisive impact of photonics in the design and development of coherent MIMO radars with widely separated antennas.

\section{Conclusions and Perspectives}

In this paper, a coherent MIMO radar network demonstrator based on photonic technology has been presented and tested in a real outdoor environment. Two cooperative closely-spaced targets have been considered and successfully resolved in cross-range by applying coherent MIMO processing. The superiority of such an approach compared with the non-coherent approach is apparent, thus demonstrating the validity of the proposed architecture. The results obtained during this preliminary campaign confirm that photonics is an enabling technology for coherent MIMO radars with widely distributed antennas, thanks not only to its capability to preserve signal coherence among the TX and RX elements, but also to its granted largebandwidth long-range undistorted signal distribution over fiber. The results presented in this paper should be considered as a starting point. Further field trials are planned in the next future. They will guide us to the development of a more complex coherent radar network, able to deploy a significantly larger number of TX and RX antennas, and specifically tailored to realtime surveillance applications.

\section{Acknowledgements}

This work has been partially supported by the EU project ROBORDER (\#641388) and NATO SPS SOLE project.

Moreover, the Authors would like to sincerely thank Dr. Carlo Noviello, from IREA-CNR, Naples, Italy, for his kind and precious help during the experimental campaign.

\section{References}

[1] A.M. Haimovich et al, "MIMO radar with widely separated antennas", IEEE Signal Processing Magazine, vol. 25, no. 1, pp. 116-129, Jan. 2008.

[2] L. Lembo et al, "Analysis of a Coherent Distributed MIMO Photonics-Based Radar Network", Proc. of the $15^{\text {th }}$ European Radar Conference (EuRAD), Madrid, Spain, Sept. 2018.

[3] G. Serafino, et al., "Phase and amplitude stability of EHF-band radar carriers generated from an active mode-locked laser," Journal of Lightwave Technology, vol. 29, no. 23, Dec. 2011.

[4] P. A. Williams, et al., "High-stability transfer of an optical frequency over long fiber-optic links," Journal of Optical Society of America, B 25, pp. 1284-1293, 2008.

[5] P. Ghelfi et al, "A fully photonics-based coherent radar system," Nature, vol. 507, March 2014.

[6] F. Laghezza et al, "Field Evaluation of a photonics-based radar system in a maritime environment compared to a reference commercial sensor," IET Radar, Sonar \& Navigation, vol. 9, no. 8, pp. 1040-1046, Oct. 2015.

[7] J. Fu, et al., "A fiber-distributed bistatic ultra-wideband radar based on optical time division multiplexing”, 2015 Int. Topic. Meet. on Microwave Photonics (MWP), Paphos, Cyprus, 2015.

[8] F. Zhang, et al., "Photonics-based MIMO radar with high-resolution and fast detection capability", Optics Express, vol. 26, no. 13, pp. 17529-17540, 2018.

[9] L. Lembo, et al., "In-Field Demonstration of a Photonic Coherent MIMO Distributed Radar Network," Proc. of IEEE Radar Conference 2019, Boston (MA), USA (submitted).

[10] G.A. Richards, "Fundamentals of Radar Signal Processing", 2nd Ed., McGraw-Hill, 2014.

[11] I. Pasya et al, "Detection Performance of M-Sequence-Based MIMO Radar Systems Considering Phase Jitter Effects," IEEE Phased Array Systems \& Technology Symp., Oct. 2013.

[12] N.H. Lehmann et al, "High Resolution Capabilities of MIMO Radar," Proc. of the $40^{\text {th }}$ IEEE Asilomar Conf. on Signals System and Computers, Pacific Groove, CA, USA, Oct.-Nov. 2006. 